\title{
Efeitos da Radiação Ionizante Sobre Comportamentos Mantidos por Contingências Operantes ${ }^{1}$
}

\author{
Lincoln da Silva Gimenes ${ }^{2}$ e Laércia Abreu Vasconcelos \\ Universidade de Brasília
}

\begin{abstract}
RESUMO - Pesquisas de laboratório com animais são tentativas para suprimir lacunas deixadas pelas metodologias utilizadas nos estudos com humanos sobre os fenômenos comportamentais que acompanham uma exposição à radiação. Neste trabalho, tratamos de estudos sobre comportamentos mantidos por diferentes tipos de contingências operantes e de variáveis radiogênicas como a dosagem de radiação. Os efeitos observados com esquemas simples de reforçamento mostram redução nas taxas de resposta. Esses efeitos são dose-dependentes e interagem com outras variáveis tais como o tipo de linha de base, taxa de respostas na linha de base e tipo de estímulo reforçador. Além disso, em esquemas concorrentes os efeitos são seletivos, segundo os componentes do esquema. Dados obtidos em procedimentos de aquisição repetida indicam uma interação da radiação com o tipo de tarefa em execução. As implicações dos estudos com animais para a compreensão das manifestações comportamentais observadas em indivíduos expostos a eventos radioativos são discutidas.
\end{abstract}

Palavras-chave: radiação ionizante; contingências operantes; toxicologia comportamental.

\section{Effects of Ionizing Radiation Upon Behaviors Maintained by Operant Contingencies}

\begin{abstract}
Laboratory animal research are attempts to fill some gaps left by the methodology used with humans about behavioral phenomena that follow radiation exposure. This work deals with studies about behaviors maintained by different operant contingencies and radiogenic variables such as radiation dosage. The characteristic effects observed under simple reinforcement schedules show a reduction in response rates. These effects are, however, dose-dependent and interact with other variables such as type of baseline, response rate during baseline and type of reinforcer. Furthermore, under concurrent schedules the effects are selective according to the schedule components. Data obtained with repeated acquisition procedures indicate also an interaction of radiation with the type of task involved. Implications of laboratory animal studies for the understanding of observed behavioral manifestations in individuals exposed to radioactive events are discussed.
\end{abstract}

Key words: ionizing radiation; operant contingencies; behavioral toxicology.

"Radiação pode ser definida como uma forma de energia, emitida por uma fonte e que se propaga de um ponto a outro sob forma de partículas com ou sem carga elétrica, ou ainda sob forma de ondas eletromagnéticas" (Okuno, 1988, p. 12). Portanto, radiação é a propagação de energia através do espaço ou da matéria. A radiação corpuscular é constituída de partículas subatômicas tais como elétrons, prótons, neutrons e alfas em altas velocidades, enquanto as radiações eletromagnéticas (fótons) não possuem massa. Radiações eletromagnéticas são todas as radiações que possuem oscilações de campos elétricos e magnéticos e cuja propagação de ondas viaja à velocidade da luz e diferem somente pela frequiência de emissão, ou seja, quanto maior a freqüência maior será a energia associada à radiação (Hendee, 1996; Scaff, 1997).

A radiação ionizante, um exemplo de radiação eletromagnética, ao atravessar a matéria produz pares de íons (cargas positivas e negativas). As radiações carregadas eletrica-

1 Trabalho parcialmente financiado pelo CNPq.

2 Endereço: Universidade de Brasília, Instituto de Psicologia, Departamento de Processos Psicológicos Básicos. Campus Universitário Darcy Ribeiro. CEP: 70910-900 Brasília - DF. E-mail: lgimenes@unb.br; laercia@unb.br mente, como, por exemplo, o feixe de elétrons ou prótons, são denominadas diretamente ionizante e as radiações eletricamente neutras são denominadas indiretamente ionizante, por exemplo, o feixe de fótons ou nêutrons (Johns, 1983; Scaff, 1997). Em nível celular, a radiação ionizante quebra as moléculas, gerando radicais livres que alteram a homeostase e interferem no metabolismo, podendo gerar morte celular, além de quebras cromossômicas. Assim, a exposição do ser humano e outros animais à radiação, que pode resultar na absorção parcial ou total de energia pelos tecidos ou órgãos, é um objeto de estudo muito importante para prevenir ou reduzir efeitos resultantes da mesma.

As pesquisas sobre alterações comportamentais que podem ocorrer em indivíduos após uma exposição à radiação ionizante são, em grande parte, baseadas em medidas de autorelato. Cada acidente radioativo é representado por um conjunto específico de variáveis e, associado a esta especificidade, está a falta de uma linha de base do indivíduo ou da comunidade afetada por este tipo de evento. As ocorrências desses eventos, por serem na maioria das vezes imprevisíveis, não permitem estudos prospectivos, além do que, questões éticas imperiosas excluem a possibilidade de estudos manipulativos paramétricos com sujeitos humanos, nessa área. Dessa forma, as pesquisas de laboratório com animais 


\section{S. Gimenes e L. A. Vasconcelos}

são tentativas importantes de suprimir algumas lacunas deixadas pelas metodologias mais tradicionais, e de aumentar nosso conhecimento sobre o fenômeno comportamental que acompanha a exposição à radiação.

Em geral, as pesquisas realizadas com sujeitos não-humanos têm se dedicado, em grande parte, à análise dos efeitos fisiológicos provocados pela radiação ionizante. Uma das principais conseqüências da radiação é o dano provocado no sistema hematopoético, acarretando na perda de células sangüíneas funcionais. Isso enfraquece o sistema imunológíco do organismo, tornando-o mais sensível a uma variedade de doenças, podendo, eventualmente, levá-lo à morte. Esses efeitos são dose-dependentes, podendo ser reversíveis após doses consideradas baixas e moderadas (doses subletais), ou irreversíveis, após doses altas (doses letais). Dose letal, geralmente expressa por $\mathrm{LD}^{50 / 30}$, é definida como a dose que em um período de 30 dias levará a óbito $50 \%$ dos indivíduos expostos à mesma. A letalidade da dose varia de espécie para espécie, sendo o rato, por exemplo, mais radiorresistente do que o macaco Rhesus e do que o homem. A dose letal média é de aproximadamente $9,0 \mathrm{~Gy}^{3}$ para o rato, 6,0 Gy para o macaco Rhesus e, 4,5 Gy para o homem.

Existem, no entanto, diferenças individuais quanto à letalidade da dose. Por exemplo, um dos indivíduos expostos ao Césio-137 durante o acidente ocorrido em Goiânia, em 1987, recebeu uma dose aproximada de 7 Gy e apresentou uma sobrevida de sete anos. Esse fato chamou a atenção de um grande número de técnicos em radiação em todo o mundo. E ainda existe uma discussão se o óbito desse indivíduo foi realmente relacionado com os efeitos da radiação no seu organismo.

Os estudos sobre os efeitos comportamentais da radiação, adaptando uma sugestão de Mickley, Bogo, Landauer e Mele (1988), podem ser classificados em dois grupos: o primeiro, dedicado ao estudo de comportamentos naturais e, o segundo, dedicado ao estudo de comportamentos que envolvem algum tipo de aprendizagem. No primeiro grupo, estariam comportamentos como atividade locomotora espontânea, agressão e consumo de alimentos e água. Em geral, e pelo menos em roedores, exposição à radiação tende a produzir decréscimos em todos esses comportamentos, isto é, uma diminuição na atividade geral do animal, um aumento no limiar a estímulos evocadores de respostas agressivas e uma redução na quantidade de alimento e água consumidos. Esses efeitos, quando produzidos por doses subletais, são dose-dependentes e transitórios. Com essas doses, os efeitos depressivos nessas atividades não aparecem de imediato. As mudanças comportamentais observadas desenvolvem-se ao longo de alguns dias, após as quais, ocorre um retorno gradativo a taxas da linha de base. Por outro lado, quando altas doses são aplicadas (doses letais), surge um fenômeno

3 A energia depositada pela radiação ionizante por unidade de massa é denominada dose absorvida e sua unidade no Sistema Internacional de Unidades e Medidas (SI) é o Gy (Gray) que substitui uma outra unidade muito utilizada no passado, o $\operatorname{rad}(\mathbf{1} \mathrm{Gy}=100 \mathrm{rad})$. designado incapacitação transitória inicial. Isto é, imediatamente após a irradiação, observa-se um decréscimo no desempenho do animal, com uma duração aproximada de 20 a 30 minutos, seguido de uma recuperação a taxas próximas à linha de base. Entretanto, devido à letalidade da dose, dentro de alguns dias ou horas, o animal apresenta redução generalizada de comportamentos, seguida de morte (Bogo, Franz \& Young, 1987).

No segundo grupo de estudos, estariam os comportamentos mantidos por esquemas de reforçamento positivo ou negativo, desempenho em discriminações complexas, desempenho em tarefas de labirinto e aversão condicionada. Apesar do vasto conhecimento acumulado sobre comportamentos mantidos por esquemas de reforçamento, poucos estudos têm avaliado os efeitos da radiação ionizante sobre esse tipo de comportamento. Entre os esquemas de reforçamento positivo estudados estão Razão Fixa (FR), Intervalo Fixo (FI) e Intervalo Variável (VI) (Gimenes, Vasconcelos \& Goulart, 1991;Jarrard, 1963; Mele, Franz\& Harrison, 1988). Esquemas de FR também foram estudados com reforçamento negativo (Mele, Franz \& Harrison, 1990). Esses estudos envolvendo contingências de reforçamento têm utilizado ratos como sujeitos experimentais.

Os procedimentos utilizados nesses estudos têm sido basicamente aqueles também utilizados em farmacologia comportamental (cf. Seiden \& Dykstra, 1977). Após a obtenção de uma linha de base estável, os sujeitos são expostos à radiação e as possíveis alterações comportamentais resultantes são analisadas. Entre as variáveis radiogênicas estudadas estão a dosagem de radiação (dentro dos limites subletais que permitem o estudo dos organismos a longo prazo), repetições de exposição e fracionamento da dose.

Os efeitos característicos até agora encontrados, com esquemas de reforçamento positivo, mostram uma redução na taxa de resposta (usualmente pressão à barra), em geral ocorrendo maximamente durante o período de 24 a 72 horas após a exposição à radiação, seguindo-se de uma recuperação gradual a taxas da linha de base. Essa recuperação ocorre em torno de 20 a 30 dias. A Figura 1 exemplifica esse padrão comportamental que se segue à radiação.

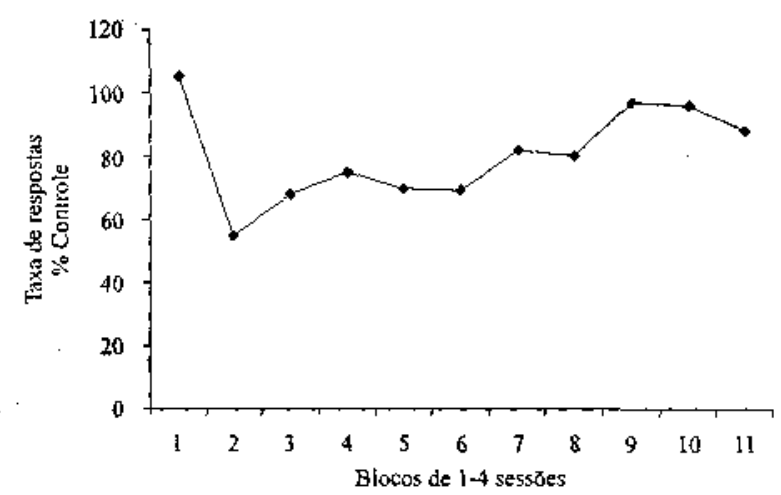

Figura 1. Efeitos de 6,5 Gy de radiação gama sobre taxa de respostas em FI $120 \mathrm{seg}$. O bloco 1 representa a sessão cinco minutos após a exposição. Os blocos subseqüentes representam a média de três sessões com exceção do bloco 7 que representa a média de quatro sessões. Esses dados são dados médios de cinco sujeitos (Adaptado de Mele \& cols., 1988). 
Entretanto, esses efeitos além de serem dose-dependentes interagem com outras variáveis tais como o tipo de linha de base, taxa de respostas na linha de base e tipo de estímulo reforçador. Mele e cols. (1988), comparando os efeitos da radiação gama gerada por Cobalto 60 (Co 60) sobre comportamentos mantidos por esquemas de reforçamento de FR 50 e FI 120 s (utilizando leite como reforço), encontraram os seguintes resultados: enquanto o desempenho em FR 50 foi afetado por exposição a 4,5 Gy, o desempenho em FI 120 s só foi afetado por 6,5 Gy, mostrando, assim, radiossensibilidade diferencial dos esquemas utilizados como linha de base. $\mathrm{O}$ estudo de Mele e colaboradores demonstrou, também, que os efeitos comportamentais de doses agudas moderadas de radiação não são cumulativos, quando se repetem as doses após o desempenho haver retornado às taxas da linha de base. Nesse estudo, os sujeitos foram expostos três vezes à mesma dose aguda de radiação com intervalos de 43 dias entre as exposições. Após cada uma das exposições, os efeitos foram semelhantes, não havendo efeitos cumulativos (pelo menos com esse número de repetições).

Gimenes e cols. (1991) compararam os efeitos da radiação gama por Co 60 sobre desempenhos em esquemas de reforçamento em FI 120 s e VI 120 s, utilizando ração em pó como reforço. Os dados obtidos demonstraram efeitos semelhantes aos obtidos por Mele e cols. (1988). Os efeitos sobre o desempenho em FI foram, entretanto, observados com doses mais baixas, de 3,5 Gy. Por outro lado, os efeitos sobre o desempenho em Intervalo Variável, com essa mesma dose, parece ter sido seletivo em relação às taxas de resposta na linha de base. Com esse esquema de reforçamento, somente altas taxas de respostas foram afetadas.

Vasconcelos, Gimenes, Magalhães, Santos, Macedo, Brandão e Goulart (1993) conduziram um outro experimento delineado para investigar a interação entre taxas de resposta em VI e os efeitos da radiação. Nesse estudo, dois grupos de ratos foram submetidos a um esquema de reforçamento em VI 120 s. Após estabilização dessa linha de base, um dos grupos foi exposto a uma sessão de radiação placebo $(0$ Gy - grupo controle) e o outro a uma sessão de radiação de 6 Gy (grupo experimental). Como pode ser observado na Figura 2, os resultados mostraram uma acentuada redução na taxa de respostas do grupo experimental, com a maior redução ocorrendo no terceiro dia após a radiação e com recuperação já a partir do quinto dia após a radiação.

Uma análise subseqüente desses dados foi realizada dividindo-se o grupo experimental em dois subgrupos diferenciados pelos níveis de taxa de respostas de cada um (denominados taxa alta e taxa baixa). Os dados mostraram mais uma vez os efeitos diferenciais da radiação em relação à taxa de respostas na linha de base. Como pode ser observado na Figura 3, apesar dos efeitos nos dois subgrupos representarem uma mesma função, a redução na taxa de respostas observada no grupo taxa alta foi mais que duas vezes superior àquela observada no grupo taxa baixa.

Esse efeito seletivo da radiação já havia sido sugerido por Mele e cols. (1988) na interpretação das diferenças encontradas entre os esquema de FR e FI. Uma análise dos

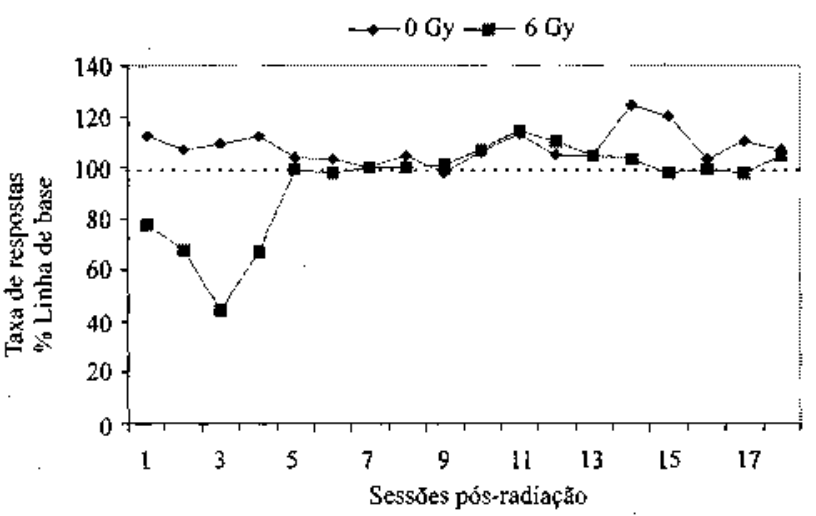

Figura 2. Taxa média de respostas nas sessões pós-radiação, para o grupo controle (0 Gy) $(n-6)$ e para o grupo experimental (6 Gy) $(n=8)$, em um procedimento de Intervalo Variável de $120 \mathrm{~s}$ (Vasconcelos \& cols., 1993

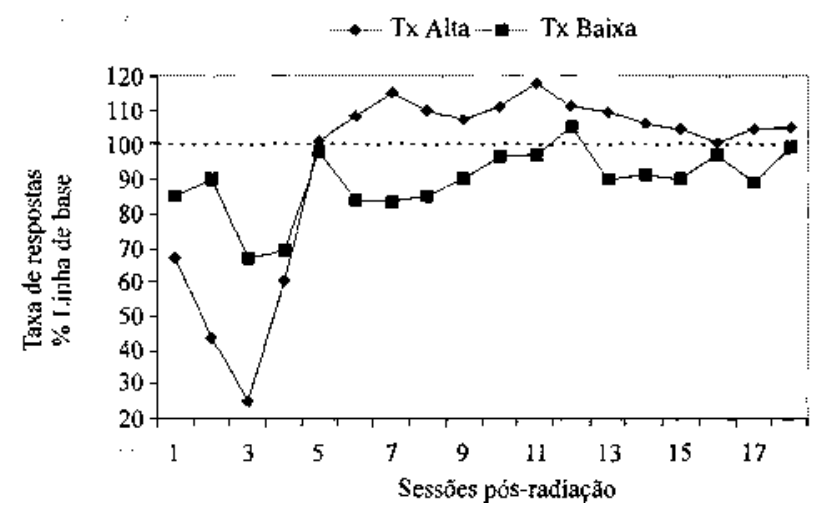

Figura 3. Taxa média de respostas nas sessões pós-radiação, para os dois subgrupos experimentais, taxa alta $(n=4)$ e taxa baixa $(n=4)$. (Vasconcelos \& cols., 1993)

desempenhos em FR e FI, por meio da inspeção de registros cumulativos, mostrou que a redução nas taxas de resposta deu-se pelo aumento das pausas pós-reforço. Os padrões de resposta não foram afetados; as corridas (jorros de respostas) observadas em FR e as acelerações positivas (scallops) observadas em FI foram mantidas.

Os efeitos observados utilizando esquemas de reforçamento negativo (comportamento de fuga) apresentam as mesmas características gerais daqueles encontrados na utilização de esquemas de reforçamento positivo. A magnitude da dose necessária para produzir os efeitos e o curso temporal desses efeitos são, no entanto, diferentes. Mele e cols. (1990), comparando o desempenho em um esquema de reforçamento negativo em FR 20 (utilizando um choque de 0,9 mA como estímulo reforçador negativo) com o desempenho em um esquema de reforçamento positivo em FR 20 (utilizando leite como estímulo reforçador positivo), encontraram as seguintes diferenças: enquanto no esquema de reforçamento positivo os efeitos apareceram com doses de 4,5 Gy, o comportamento de fuga só foi afetado com doses de 7,5 Gy; o efeito apareceu mais tardiamente para o comportamento de fuga do que para o comportamento mantido pelo estímulo reforçador positivo; a recuperação à taxas de linha de base deu-se mais lentamente para o comportamento 


\section{S. Gimenes e L. A. Vasconcelos}

de fuga que para o comportamento mantido pela consequiência positiva (o efeito demorou mais a ocorrer, mas uma vez ocorrido permaneceu por um tempo mais longo).

Os efeitos descritos acima, tanto em contingências positivas quanto negativas, são observados quando a radiação ocorre de forma aguda. Comparações utilizando-se as mesmas doses de forma aguda ou fracionada (por exemplo 7,5 Gy em uma única exposição ou cinco exposições de 1,5 Gy com intervalos de algumas horas entre as mesmas) demonstraram que os efeitos só ocorrem no primeiro caso (Mele \& col., 1988; 1990). O fracionamento da dose, assim como a repetição da dose aguda após um período de recuperação não produziram efeitos cumulativos.

O tipo de estímulo reforçador parece ser, também, uma variável importante na determinação dos efeitos produzidos pela radiação. Comparando alguns dos estudos, pode-se traçar uma curva representativa da relação entre doses de radiação e os diferentes estímulos reforçadores utilizados. A Figura 4 apresenta essa relação entre tipos de reforçadores e a menor dose de radiação ionizante necessária para produzir um efeito sobre as taxas de respostas. Entretanto, devido às diferenças entre os estudos, essa curva exemplifica a possível relação entre as variáveis e serve como sugestão para a investigação sistemática dessa relação.

Como citado anteriormente, um dos efeitos produzidos pela radiação ionizante é o decréscimo observado no consumo de alimentos e água. Entretanto, esse efeito parece ocorrer somente em situações de alimentação livre. Nos estudos realizados com esquemas de reforçamento em FI, apesar de ter havido redução nas taxas de resposta, não houve redução na obtenção dos reforços programados. O efeito seletivo sobre o desempenho em VI pode, também, ser relacionado com a obtenção dos reforços. Essas diferenças sugerem propriedades reforçadoras diferentes para alimentação livre em comparação com alimentação obtida por meio de contingências operantes, ou ainda, sugerem que os procedimentos de privação utilizados nessas contingências podem minimizar o efeito da radiação observado na alimentação livre.

A obtenção dos reforços programados mesmo com a redução na taxa de respostas aponta para a distribuição de re-

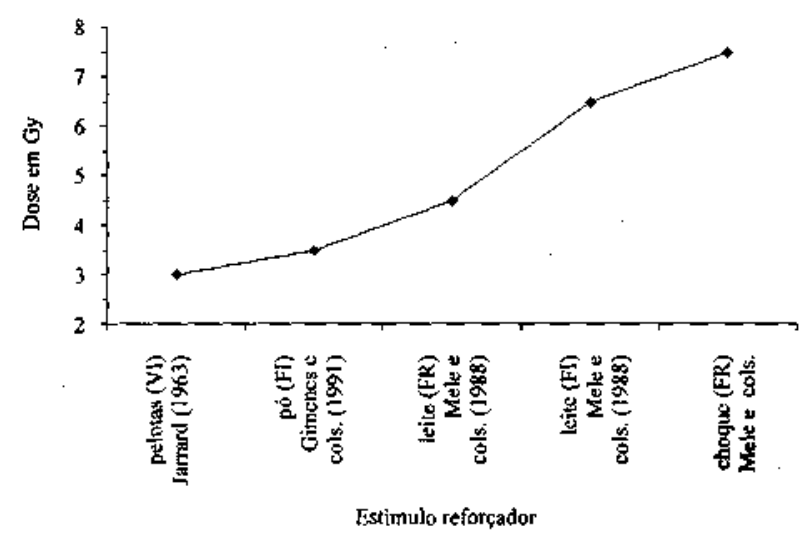

Figura 4. Relação entre dose de radiação e tipo de estímulo reforçador e esquemas de reforçamento utilizados em diferentes estudos. Os valores representam a menor dose que produziu um efeito sobre as taxas de respostas. forços como uma das possíveis variáveis interagindo com os efeitos da radiação. Para verificar essa possibilidade, Vasconcelos e cols. (1995) realizaram um outro experimento manipulando a distribuição de reforços por meio de um esquema concorrente de reforçamento em VI 30 s VI 120 s, com pelotas de alimento como reforço. Após estabilização na distribuição de respostas e de reforços, os sujeitos foram expostos a uma sessão de irradiação placebo (0 Gy), a uma sessão de irradiação de 4,5 Gy, ou a uma sessão de irradiação de 6,0 Gy por Co 60. Os dados demonstraram uma redução nas taxas de respostas dos sujeitos expostos ao Co 60 em relação aos sujeitos expostos ao placebo. Após a irradiação, houve uma maior redução na taxa de respostas associada com o esquema de menor densidade de reforços, no caso VI 120 s. Essa alteração refletiu na distribuição de respostas com um aumento relativo das respostas associadas ao esquema de maior densidade de reforços, no caso VI $30 \mathrm{~s}$.

Pode ser observado na Figura 5 que as alterações quer na taxa, quer na distribuição de respostas não alteraram a distribuição de reforços programados. O esquema com maior intervalo entre reforços tende a ser menos afetado pela redução de respostas; a não ser quando a redução de repostas é muito grande, a relação entre reforços programados e reforços obtidos mantém-se inalterada. Isso ocorre pelo fato das contingências de intervalo requererem apenas uma resposta ao final do intervalo para a apresentação do reforço. Com intervalos pequenos entre reforços, o espaçamento das respostas (taxas mais baixas) pode provocar um alongamento nos intervalos, o que reflete em uma diminuição no total de reforços obtidos nas sessões, quando as mesmas têm um tempo fixo de duração. Por outro lado, taxas baixas têm uma maior probabilidade de atender mais prontamente as exigências da contingência quando os intervalos entre reforços são longos, o que mantém a relação entre reforços programados e obtidos praticamente inalterada.

Os efeitos comportamentais até aqui apresentados foram observados em estados estáveis. Informações com respeito aos efeitos sobre a aquisição do comportamento ainda são escassas. Um dos procedimentos que melhor se ajusta a esse objetivo é o de aquisição repetida. Nesse procedimento, uma nova seqüência de respostas deve ser aprendida a cada sessão experimental, isto é, aprendizagem ocorre a cada sessão. Além da avaliação da aprendizagem intra-sessão, a aprendizagem intersessões pode, também, ser observada por meio das alterações no padrão de aprendizagem com o decorrer do número de sessões. Geralmente, o padrão de aprendizagem observado nesse procedimento é comparado ao padrão observado em outro procedimento designado desempenho, onde uma mesma seqüência é mantida em todas as sessões. Em um estudo preliminar, após 60 sessões de linha de base em um procedimento de esquema múltiplo de aquisição repetida de uma sequiência de três respostas e de desempenho, ratos foram expostos a uma sessão de radiação de 4,5 Gy produzida por um acelerador linear (Vasconcelos, Gimenes, Brandão, Tubino, Nogueira \& Caballero, 1997). Os dados apresentados na Figura 6 mostram os efeitos típicos da redução na taxa de respostas, tanto no componente de aquisição repetida quanto no de desempenho. 

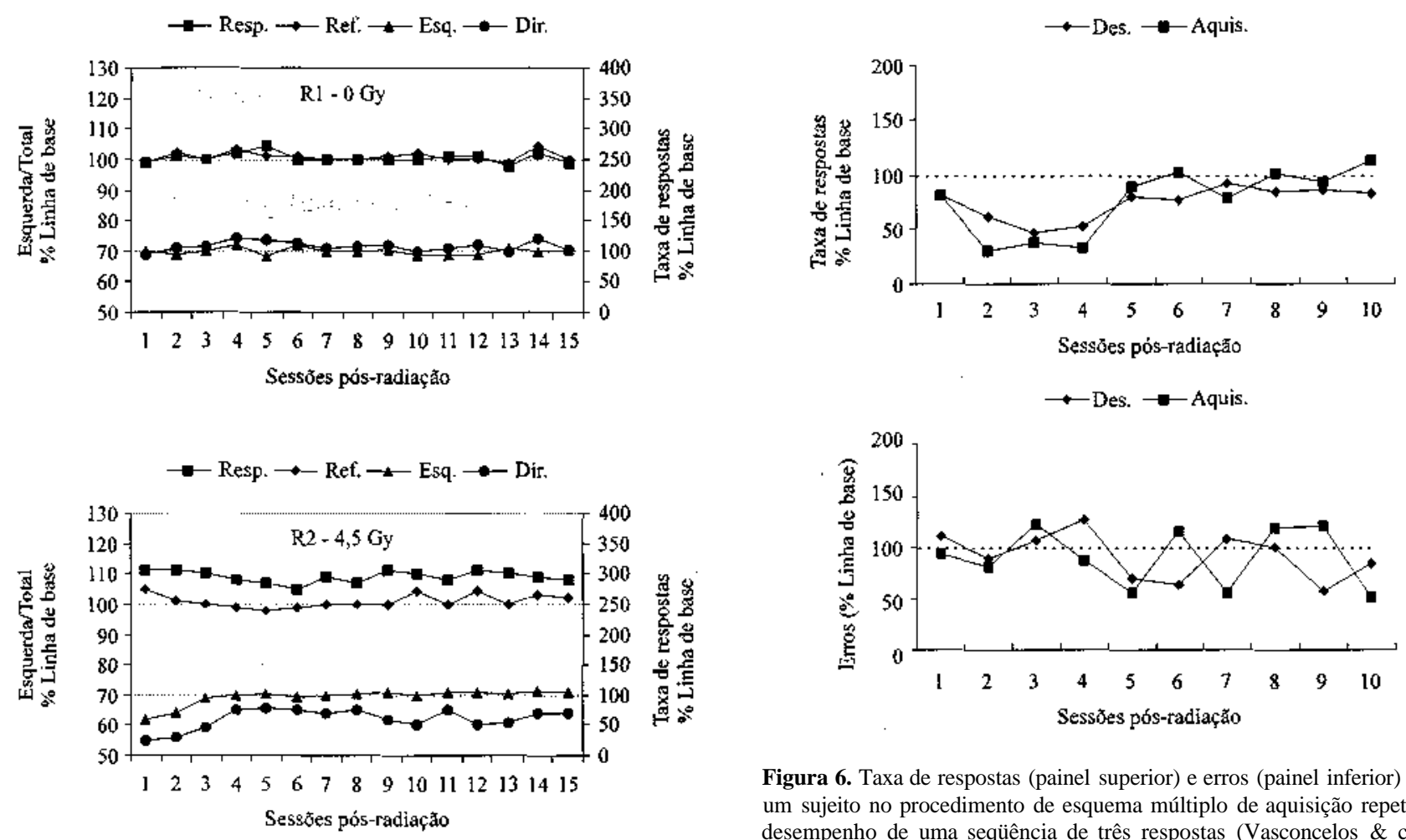

Figura 6. Taxa de respostas (painel superior) e erros (painel inferior) para um sujeito no procedimento de esquema múltiplo de aquisição repetida / desempenho de uma sequiência de três respostas (Vasconcelos \& cols. 1997).

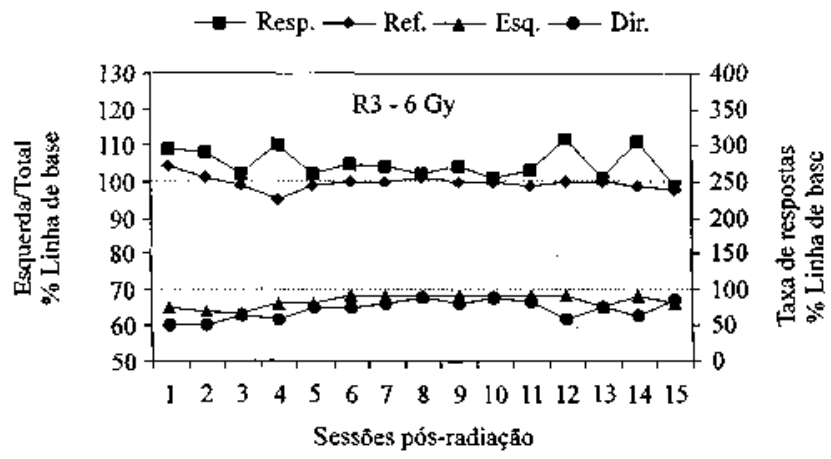

e possível redução na obtenção de reforços e, conseqüentemente, menor consumo. Entretanto, esses efeitos anoréxicos e letárgicos interagem com os parâmetros de diferentes contingências, podendo ser potencializados ou atenuados pelos mesmos.

Em um outro nível explicativo, os efeitos comportamentais da radiação parecem ser modulados pelo sistema dopaminérgico. Em um estudo realizado por Cory-Slechta, Pazmino e Bare (1997), reduções nos níveis de dopamina foram seguidas de reduções nas taxas de respostas em um esquema de FI. Mais recentemente, Foulon, Lalouette, Lambert, Martin, Fatome e Martin (1999) observaram que a exposição à radiação ionizante produziu uma diminuição nos níveis de dopamina e seus metabólicos. Além disso, o sistema dopaminérgico desempenha importante papel sobre o funcionamento do sistema gastrintestinal. Tomados em conjunto, esses dados são evidências que apontam para o sistema dopaminérgico como um dos moduladores dos efeitos da radiação ionizante sobre o comportamento.

Em resumo, os dados até aqui obtidos mostram que os efeitos da radiação ionizante sobre comportamentos mantidos por contingências operantes são dose-dependentes e transitórios (com doses subletais). Além disso, a intensidade dos efeitos interagem com outras variáveis, tais como, tipo de linha de base e padrão comportamental observado nas mesmas, tipo de estímulo reforçador utilizado e complexidade das tarefas exigidas pelas contingências.

Como afirmado no início desse trabalho, o estudo dos efeitos da radiação ionizante sobre o comportamento humano, diferentemente de outros agentes tóxicos como o chumbo, por exemplo, depende da ocorrência de acidentes 


\section{S. Gimenes e L. A. Vasconcelos}

imprevisíveis e indesejáveis. Dessa forma, os estudos com animais em laboratório nos permitem acumular um conhecimento necessário para que possamos compreender as relações entre esse tipo de radiação e comportamento e, assim, nos prepararmos para possíveis situações onde a avaliação dessas relações se fizer necessária. Além disso, o estudo dos efeitos comportamentais da radiação com indivíduos envolvidos em um acidente radioativo é extremamente complexo e difícil, tendo em vista as profundas alterações sociais que um evento dessa natureza pode ocasionar, resultando em uma multideterminação das mudanças comportamentais observadas.

Em um estudo realizado com indivíduos envolvidos no acidente com Césio-137 na cidade de Goiânia, foram detectadas pelo menos três possíveis fontes responsáveis pela manutenção a longo prazo de níveis de ansiedade e queixas de problemas de saúde (registrados como comportamentos verbais por meio de instrumentos padronizados para esse fim) (Gimenes \& Vasconcelos, 1997). A primeira dessas fontes advém do desconhecimento sobre os possíveis danos que podem ser causados pela radiação, a médio e longo prazos. A incerteza sobre o estado futuro da saúde desses indivíduos e a de seus familiares parece contribuir para um constante estado de ansiedade. Tal incerteza pode, também, contribuir para a atribuição à radiação de qualquer alteração nos seus estados de saúde. Uma segunda fonte refere-se ao controle de estímulos exercido pelo longo processo judicial e pelas freqüentes incursões da mídia contribuindo para a manutenção viva das lembranças do evento e, conseqüentemente, dos estados emocionais a ele associados. Como terceira fonte, estariam as conseqüências sócio-econômicas relacionadas com o envolvimento no acidente. Compensação financeira, atenção social e atenção médica dispensada a esses indivíduos podem ter contribuído para legitimizar as queixas de saúde e de ansiedade apresentadas pelos mesmos. As observações realizadas nos estudos com animais, que mostram a transitoriedade dos efeitos comportamentais da radiação, bem como a modulação desses efeitos por parâmetros das contingências contribuem para a interpretação de que os comportamentos verbais de relatos de ansiedade e problemas de saúde, que inicialmente podem ter sido produzidos pelos parâmetros da radiação, passaram a ser mantidos por contingências sociais decorrentes do evento radioativo.

Assim, os resultados obtidos com as pesquisas utilizando animais, junto às observações dos indivíduos envolvidos em um acidente radioativo sugerem que variáveis motivacionais, tanto na dimensão biológica (e.g., privação e qualidade do reforço), quanto na social (e.g., atenção social e desconhecimento sobre riscos futuros), desempenham um importante fator na determinação dos efeitos comportamentais da radiação ionizante, quer em animais de laboratório, quer em sujeitos humanos.

A apresentação de alguns aspectos dos efeitos da radiação ionizante sobre o comportamento mantido por contingências operantes e o papel desempenhado por algumas variáveis não pretende esgotar o assunto. Outras variáveis podem, também, interagir diferentemente com esses compor- tamentos. Entre elas estão os diferentes tipos de radiação e os diferentes tipos de tarefas que requerem diferentes níveis de complexidade do comportamento sob estudo. Essas questões continuam ainda abertas ao escrutínio empírico.

Finalmente, a utilização cada vez maior da energia nuclear, o que infelizmente torna mais provável acidentes radioativos, requer conhecimentos cada vez mais aperfeiçoados sobre os efeitos da radiação. Esses efeitos sobre o comportamento são ainda relativamente escassos, o que demanda estudos adicionais sobre esse fenômeno. Dadas as características desse objeto de estudo, pesquisas com animais ainda são a fonte mais precisa de produção dos conhecimentos necessários a uma preparação científica adequada; que viabilize programas para a compreensão dos efeitos da radiação bem como para intervenção tanto preventiva quanto após a ocorrência de eventos radioativos. As pesquisas com animais nessa área, como em todas as áreas que oferecem risco aos sujeitos, devem, entretanto, seguir sempre as normas determinadas pelos comitês de ética na pesquisa, nos seus diferentes níveis de atuação ${ }^{4}$.

\section{Referências}

Bogo, V., Franz, C.G., \& Young, R.W. (1987). Effects of radiation on monkey visual discrimination performance. Em E.M. Fielden, J.F. Fowler, J.H. Hendry, \& D. Scott (Orgs.), Radiation research. Proceedings of the ${ }^{\text {th }}$ International Congress on Radiation Research (Volume 1). London: Taylor and Francis.

Cory-Slechta, D.A., Pazmino, R. \& Bare, C. (1997). The critical role of nucleus accumbens dopamine system in the mediation of fixed interval schedule-controlled operant behavior. Brain Research, 764, 253-256.

Foulon, O., Lalouette, F, Lambert, F, Martin, S. Fatome, M. \& Martin, C. (1999). Effects of neutron-gamma radiation on dopamine and seratonin metabolism in the rat brain: A regional analysis. Journal of Neuroscience Research, 55, 770-775.

Gimenes, L.S. \& Vasconcelos, L.A. (1997). Los desastres radiactivos y sus efectos a largo plazo: El caso Césio-137. Desastres \& Sociedad, 8, 55-62.

Gimenes, L.S., Vasconcelos, L.A. \& Goulart, F.B. (1991). Effects of ionizing radiation on operant performance by rats under interval schedules of reinforcement [Resumo]. Em Association for Behavior Analysis (Org.), Procedings of the $17^{\text {th }}$ Annual Convention of the Association for Behavior Analysis (p. 264). Atlanta: ABA.

Hendee, W.R. (1996). Radiation Therapy Physics. St. Louis: Mosby.

Jarrard, L.E. (1963). Effects of X-irradiation on operant behavior in the rat. Journal of Comparative and Physiological Psychology, 56,608-611.

Johns, H.E. (1983). The Physics of radiology. Springfield: Charles C. Thomas.

4 Os animais utilizados nos experimentos descritos neste trabalho foram mantidos e manipulados segundo os princípios enunciados pelo Guide for the Care and Use of Laboratory Animals (National Research Council, (1996). 
Mele, PC, Franz, CG. \& Harrison, J.R. (1988). Effects of sublethal doses of ionizing radiation on schedule-controlled performance in rats. Pharmacology, Biochemistry and Behavior, 30, 1007-1014.

Mele, PC, Franz, C.G., \& Harrison, J.R. (1990). Effects of ionizing radiation on fixed-ratio escape performance in rats. Neurotoxicology and Teratology, 12, 367-373.

Mickley, G.A., Bogo, V., Landauer, M.R. \& Mele, PC. (1988). Current trends in behavioral radiobiology. Em PD. McCormack, C.E. Swenberg, \& H. Bucker (Orgs.), Terrestrial space radiation and its biological effects (pp. 517-536). New York: Plenum Publishing Corporation.

National Research Council (1996). Guide for the care and use of laboratory animals. Washington: National Academy Press.

Okuno, E. (1988). Radiação: efeitos, riscos e benefícios. São Paulo: Harbra.

Scaff, L.A.M. (1997). Física da radioterapia. São Paulo: Sarvier.

Seiden, L.S. \& Dykstra, L.A. (1977). Psychophannacology: A biochemical and behavioral approach. New York: Van Nostrand Reinhold Company.

Vasconcelos, L.A., Gimenes, L.S., Magalhães, E.R., Santos, A.C.D., Macedo, J.L., Brandão, A. \& Goulart, F.B. (1993). Efeitos de doses subletais de irradiação ionizante sobre o desempenho em intervalo variável em ratos [Resumo]. Em Sociedade Brasileira de Psicologia (Org.), Resumos de comunicações científicas, XXVII Reunião Anual de Psicologia (p. 49). Ribeirão Preto: SBP.

Vasconcelos, L.A., Gimenes, L.S., Brandão, A.M., Bezerra, A.C. A., Dacanal, J.N., Campos, L.M. \& Goulart, F.B. (1995). Irradiação ionizante e o desempenho de ratos sob esquema concorrente VI-VI [Resumo]. Em Sociedade Brasileira de Psicologia (Org.), Resumos de comunicações científicas, XXV Reunião Anual de Psicologia (p. 444). Ribeirão Preto: SBP.

Vasconcelos, L.A., Gimenes, L.S., Brandão, A.M., Tubino, C., Nogueira, T. \& Caballero, K.C.S. (1997). Desempenho e aquisição repetida de seqüências de respostas em ratos: alguns efeitos da radiação ionizante [Resumo]. Em Sociedade Brasileira de Psicologia (Org.), Resumos de comunicações científicas, XXIII Reunião Anual de Psicologia (p. 484). Ribeirão Preto: SBP.

Winsauer, P.J., Verrees, J.F., O'Halloran, K.P., Bixler, M.A. \& Mele, PC. (1994). Effects of chlordizepoxide, 8-OH-DPAT and ondansetron on radiation-induced decreases in food intake in rats. The Journal of Pharmacology and Experimental Therapeutics, 270, 142-149. 